\title{
Formulation and Evaluation of Chitosan-Based Ampicillin Trihydrate Nanoparticles
}

\author{
Partha Saha ${ }^{1}$, Amit K Goyal ${ }^{2}$ and Goutam Rath ${ }^{2^{\star}}$ \\ ${ }^{1}$ Kanak Manjari Institute of Pharmaceutical Sciences, Rourkela (Orissa), ${ }^{2}$ Department of Pharmaceutics, ISF College
} of Pharmacy, Moga (Punjab), India

\begin{abstract}
Purpose: To develop ampicillin trihydrate-loaded chitosan nanoparticles by modified ionic gelation method and evaluate their antimicrobial activity.

Methods: Ampicillin trihydrate-loaded chitosan nanoparticles were prepared by ionic gelation method with the aid of sonication. Parameters such as the zeta potential, polydispersity, particle size, entrapment efficiency and in vitro drug release of the nanoparticles were assessed for optimization. The antibacterial properties of the nanoparticle formulation were evaluated and compared with that of a commercial formulation (reference).

Results: Scanning electron microscopy revealed that the nanoparticles were in the nanosize range but irregular in shape. Concentrations of $0.35 \% \mathrm{w} / \mathrm{v}$ of chitosan and $0.40 \% \mathrm{w} / \mathrm{v}$ sodium tripolyphosphate (TPP) and a sonication time of 20 min constituted the optimum conditions for the preparation of the nanoparticles. In vitro release data showed an initial burst followed by slow sustained drug release. The nanoparticles demonstrated superior antimicrobial activity to plain nanoparticles and the reference, due probably to the synergistic effect of chitosan and ampicillin trihydrate.

Conclusion: Modified ionic gelation method can be utilized for the development of chitosan nanoparticles of ampicillin trihydrate. Polymer and crosslinking agent concentrations and sonication time are rate-limiting factors for the development of the optimized formulation. The chitosan nanoparticles developed would be capable of sustained delivery of ampicillin trihydrate.
\end{abstract}

Keywords: Ampicillin trihydrate; Chitosan; Nanoparticles; Ionic gelation method; Antibacterial activity 


\section{INTRODUCTION}

Controlled drug delivery systems offer numerous advantages over conventional dosages forms, including improved efficacy, reduce toxicity, and improved patient compliance, and can be utilized in the form of nanocarriers in drug delivery [1]. Nanoparticles are solid colloidal particles with diameters ranging from $1-1000 \mathrm{~nm}$ [2]. They consist of macromolecular materials in which the active ingredient is dissolved, entrapped, encapsulated, adsorbed or chemically attached [3]. They may be prepared from a variety of materials such as proteins, polysaccharides and synthetic polymers. The selection of materials is dependent on many factors including: (a) size of nanoparticles required; (b) inherent properties of the drug, e.g., solubility and stability; (c) surface characteristics such as charge and permeability; (d) degree of biodegradability, biocompatibility and toxicity; and (e) drug release profile desired [4]. Nanotechnologies are attractive candidates for the delivery of, amongst others, antibiotics in infections caused by bacteria.

Generally, encapsulation of antibiotics in nanoparticles increases the maximal tolerated dose and therapeutic index of antibiotics when compared with the free drug [5-7]. The entrapment of ampicillin in poly(isobutylcyanoacrylate) nanoparticles increased 120-fold the efficacy of the antibiotic in an experimental acute infection of mice by Salmonella typhimurium.

Most nanoparticles prepared from waterinsoluble polymers are subjected to heat, organic solvent or high shear force during production and this may be harmful to drug stability. Moreover, some preparation methods such as emulsion polymerization and solvent evaporation are complex, and require a number of preparation steps that are time and energy consuming. In contrast, water-soluble polymers offer mild and simple preparation methods without the use of organic solvent and high shear force. Among water-soluble polymers available, chitosan is one of the most extensively studied. This is because chitosan possesses some ideal properties of a polymeric carrier for nanoparticles such as biocompatibility, biodegradability, non-toxicity, and low cost. It possesses a positive charge and exhibits an absorption enhancing effect. This characteristic can be employed to prepare cross-linked chitosan nanoparticles. The mechanism of chitosan Chitosan nanoparticle formation is usually based on electrostatic interaction between the amine group of chitosan and a negatively charged group or polyanion such as tripolyphosphate [8]. Furthermore, it possesses antimicrobial property and adsorbs toxic metals such as mercury, cadmium, lead, etc [9]..

In the present study, chitosan nanoparticles containing ampicillin trihydrate were prepared using ionic gelation method assisted by sonication. The developed systems were characterized for size, shape, zeta-potential, entrapment efficiency and in-vitro release. They were also assessed for anti-microbial activity against Staphylococcus aureus.

\section{EXPERIMENTAL}

\section{Materials}

Ampicillin trihydrate IP was purchased from Aurobindo Pharma Ltd, Hyderabad and chitosan from Central Institute of Fisheries Technology, Cochin, India. Glacial acetic acid, beef extract powder, sodium tripolyphosphate and yeast extract powder were obtained from Merck Specialties Private Limited, Mumbai, India. All other chemicals used were of analytical grade.

\section{Preparation of nanoparticles}

Blank chitosan nanoparticles were prepared by ionic gelation method. Different concentrations of polymer, ranging from 0.10 to $0.75 \% \mathrm{w} / \mathrm{v}$, were dissolved in $1.5 \% \mathrm{v} / \mathrm{v}$ acetic acid solution. Sodium tripolyphosphate solution was also prepared in distilled water 
in concentrations ranging from 0.10 to 0.75 $\% w / v$. Sodium tripolyphosphate solution was added dropwise with a syringe to chitosan solution while stirring, followed by sonication for $20 \mathrm{~min}$. The resulting suspension was subsequently centrifuged at $15000 \mathrm{rpm}$ for 10 min. The pellets obtained were re-suspended in deionised water by sonication, centrifuged and dried at room temperature (about $25^{\circ} \mathrm{C}$.

Drug-loaded chitosan nanoparticles were formed spontaneously upon dropwise addition of $12 \mathrm{ml}$ of $0.4 \%$ aqueous sodium tripolyphosphate solution to $20 \mathrm{ml}$ of 0.35 $\% w / v$ chitosan solution containing $2-5$ $\mathrm{mg} / \mathrm{ml}$ of the drug under magnetic stirring, followed by sonication. The resulting nanoparticle suspensions were centrifuged 4 times (15 min each) at $15000 \mathrm{rpm}$, washed with distilled water and dried.

\section{Determination of zeta potential}

The zeta potential of the drug-loaded chitosan nanoparticles was measured on a zetasizer (Malvern Instruments) by determining the electrophoretic mobility in a microelectrophoresis flow cell [10-11]. All the samples were measured in water at $25^{\circ} \mathrm{C}$ in triplicate..

\section{Measurement of mean particle size}

The mean size of the nanoparticles was determined by photocorrelation spectroscopy (PCS) on a submicron particle size analyzer (Malvern Instruments) at a scattering angle of $90^{\circ} \quad$ [12]. A sample $(0.5 \mathrm{mg})$ of the nanoparticles suspended in $5 \mathrm{ml}$ of distilled water was used for the measurement.

\section{Determination of particle morphology}

Particle morphology was determined by scanning electron microscopy (Jeol JSM6480LV, NIT, Rourkela, India). Dried particles were taken in a piece of black tape and attached to the sample holder. Particle morphology was determined under low vacuum.

\section{Determination of entrapment efficiency}

The encapsulation efficiency and loading capacity of nanoparticles was determined by first separating the nanoparticles formed from the aqueous medium by ultracentrifugation at $15000 \mathrm{rpm}$ for $30 \mathrm{~min}$. The amount of free ampicillin trihydrate in the supernatant was measured by UV spectrophotometery at 257nm (Elico, BL 198, Bio Spectrophotometer). The ampicillin trihydrate entrapped in the nanoparticles was calculated as Eq 1.

Entrapment efficiency (\%) $=(\mathrm{Tp}-\mathrm{Tf}) 100 / \mathrm{Tp} \ldots$ (1) where $\mathrm{Tp}$ is the total ampicillin trihydrate used to prepare the nanoparticles and $\mathrm{Tf}$ is the free ampicillin trihydrate in the supernatant

\section{Evaluation of in vitro drug release}

The ampicillin trihydrate loaded chitosan nanoparticles, after separation by ultracentrifugation, were re-dispersed in $5 \mathrm{~mL}$ $0.2 \mathrm{~mol} / \mathrm{L}$ phosphate buffer solution PBS solution $(\mathrm{pH}$ 7.4), placed in a dialysis membrane bag, tied and immersed in $150 \mathrm{~mL}$ of PBS in a $250 \mathrm{ml}$ beaker. The entire system was stirred continuously at $37{ }^{\circ} \mathrm{C}$ with a magnetic stirrer. At pre-determined time intervals, $5 \mathrm{~mL}$ of the release medium was removed and replaced with $5 \mathrm{~mL}$ of fresh PBS solution. The amount of ampicillin trihydrate in the release medium was evaluated by UV spectrophotometry at $257 \mathrm{~nm}$.

\section{Assessment of antimicrobial activity}

Nutrient agar media ( $\mathrm{pH}$ 6.5-6.6), composed of the following ingredients, was prepared: peptone $(1.2 \mathrm{~g})$, pancreatic digest of casein $(0.8 \mathrm{~g})$, yeast extracts $(0.6 \mathrm{~g})$, beef extract $(0.3 \mathrm{~g})$, dextrose $(0.2 \mathrm{~g})$ and agar $(3 \mathrm{~g})$.

The strain selected for the study, Staphylococcus aureus, was cultured on the nutrient agar medium. The cup plate technique was used. Nutrient agar plates were prepared by pouring $20 \mathrm{ml}$ autoclaved nutrient agar into a sterile Petri dish. Four 
cups each of $8 \mathrm{~mm}$ diameter were prepared by scooping out medium with a sterilized cork borer. Prior to this, the agar was streaked with the organism. The solution of each formulation - $0.2 \mathrm{ml}$ of ampicillin trihydrate (5 $\mu \mathrm{g} / \mathrm{ml}), \quad 0.06 \mathrm{ml}$ of blank nanoparticles (without drug), $0.06 \mathrm{ml}$ of drug-loaded nanoparticles $(0.015 \mathrm{mg} / \mathrm{ml})$ and $0.025 \mathrm{ml}$ of a commercial formulation of ampicillin trihydrate $(0.04 \mathrm{mg} / \mathrm{ml}))$ - were added separately to the cups and the Petri dishes incubated for $48 \mathrm{~h}$ at $37 \pm 2{ }^{\circ} \mathrm{C}$. Zone of inhibition was determined after 12, 24 and 48 $\mathrm{h}$ to evaluate the extent of bacterial inhibition by the preparations. All the experiments were performed in triplicate.

\section{Statistical analysis}

In vitro results of different formulations were compared statistically to understand the level of significance using PAST statistical software. The results are presented as mean \pm standard deviation (S.D.). Student's t-test was used to compare mean values of different groups for the determination of antimicrobial activity. Statistical significance was set at $p<0.05$.

\section{RESULTS}

The most satisfactory nanoparticles were obtained at a chitosan concentration of 0.35 $\% w / v$ and sodium tripolyphosphate concentration of $0.40 \% \mathrm{w} / \mathrm{v}$.

\section{Entrapment efficiency}

The concentration of drug for optimal entrapment of drug in the nanoparticles was $4 \mathrm{mg} / \mathrm{ml}$. The data (see Table 1) revealed that drug entrapment efficiency was dependent on polymer concentration.

\section{Zeta potential, particle size, morphology and polydispersity}

As Table 1 shows, the size of the nanoparticles decreased after sonication while zeta potential was unchanged. Zeta potential was over $+20 \mathrm{mV}$ for all formulations while polydispersity index was less than 0.3 , which indicates homogeneous nature of the formulation. Sonication for $20 \mathrm{~min}$ reduced particle size to nano level; thereafter, there was no further change in particle size. Although the particles fell within nanosize range $(200-500 \mathrm{~nm})$ [13], they were, however, irregular in shape (see Fig. 1).

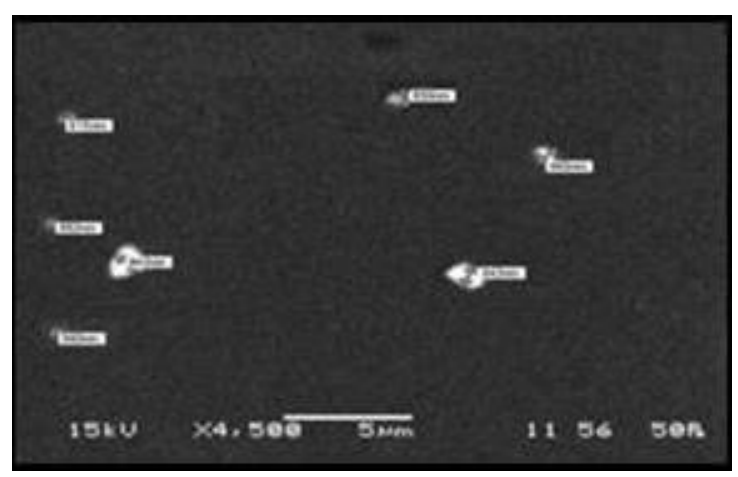

Fig 1: SEM image of ampicillin trihydrate-loaded nanoparticles

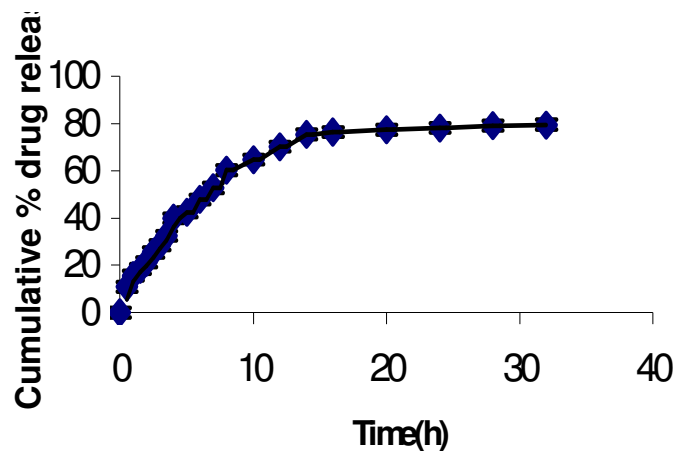

Fig 2: Drug release from ampicillin-loaded chitosan nanoparticles

\section{In vitro drug release}

The in vitro release data in Fig 2 indicate that the drug-loaded formulation obtained after sonication exhibited sustained release behavior with a steady rise in cumulative drug release (>70\%) up to the $15^{\text {th }}$ hour. Thereafter, there was no further significant release of the drug. 


\section{Antimicrobial activity}

Table 2 indicates that all the test preparations containing the drug, including the drugloaded chitosan nanoparticles, demonstrated significantly greater zone of inhibition $(p<$ 0.05) than the blank nanoparticles (i.e., without drug).

Table 2: Zone of inhibition of test samples

\begin{tabular}{lccc}
\hline \multirow{2}{*}{ Test samples } & \multicolumn{3}{c}{ Zone of inhibition (mm) } \\
\cline { 2 - 4 } & $\mathbf{1 2}^{\text {th }} \boldsymbol{h r}$ & $\mathbf{2 4}^{\text {th }} \boldsymbol{h r}$ & $\mathbf{4 8}^{\text {th }} \boldsymbol{h r}$ \\
\hline Blank & $7.5 \pm 0.1$ & $8.0 \pm 0.1$ & $8.0 \pm 0.1$ \\
Plain drug & $11.0 \pm 0.1$ & $13.0 \pm 0.2$ & $13.0 \pm 0.1$ \\
Reference drug & $11.0 \pm 0.1$ & $12.0 \pm 0.1$ & $12.0 \pm 0.1$ \\
$\begin{array}{l}\text { Drug-loaded } \\
\text { nanoparticles }\end{array}$ & $11.0 \pm 0.1$ & $11.5 \pm 0.1$ & $14.0 \pm 0.1$ \\
\hline
\end{tabular}

\section{DISCUSSION}

Nanoparticles incorporating chitosan are usually characterised by polymeric chains that are electrostatically linked as a result of ionic interactions. Moreover, nanoparticles are of great interest in pharmaceutical applications.

Chitosan is a biocompatible, biodegradable and natural polymer. It appeared to have good compatibility with the entrapped ampicillin trihydrate as there was no clear evidence of interaction between the two compounds. Thus, nanoparticles prepared by ionic gelation method using chitosan as polymer and sodium tripolyphosphate as crosslinking agent produced particles of good stability. Entrapment of the drug within the gel network of the chitosan matrix may have made the formulation more stable. It seems that the relatively lower viscosity of chitosan with a concentration as low as 0.35 $\%$ ) and an appropriate concentration of TPP $(0.4 \% \mathrm{w} / \mathrm{v})$ promoted the formation of nanoparticles. A fixed sonication time of 20 min yielded smaller and more uniform nanoparticles. This may be attributed to the fact that at this concentration, optimum viscosity was attained by chitosan solution for nanoparticle formation. Sonication time was also an important parameter for nanoparticle formation probably because sonication helped to reduce particle size.

Zeta potential of the developed formulation was in the range of $34-37 \mathrm{mV}$, which suggests that the formulation was fairly stable. The higher zeta potential indicate that chitosan nanoparticles were stable. It seems likely that the long amino groups hinder anion adsorption and keep high the value of the electrical double layer thickness, and thus preventing aggregation. The polydispersity data reinforce this assertion. Furthermore, the SEM image showed that each particle unit exhibited a nanostructure. The nanoparticles also exhibited sustained drug release behavior. While chitosan has its own antibacterial activity, it is clear that incorporation of ampicillin trihydrate in the chitosan nanoparticles produced synergism. Compared with the commercial preparation of ampicillin trihydrate (reference), the drugloaded nanoparticles exhibited similar antibacterial activity within the first $12 \mathrm{~h}$ but thereafter, showed higher activity.

\section{CONCLUSION}

This work confirms that modified ionic gelation method is useful for the development of chitosan nanoparticles. The concentration of polymer and crosslinking agent as well as sonication time are important factors in the development of ampicillin trihydrate nanoparticles. The inherent antimicrobial properties of chitosan can be exploited for the formulation of nanoparticle formulations of antimicrobial agents.

\section{ACKNOWLEDGEMENT}

We are greatful to Kanak Manjari Institute of Pharmaceutical Sciences, Rourkela, Oriss, and ISF College of Pharmacy, Moga, Punjab, both in India, for providing the required facilities for this work. 


\section{REFERENCES}

1. Majeti NV, Kumar R. Nano and microparticles as controlled drug delivery devicesJ Pharm Sci 2000; 3(2): 234-258.

2. Mohanraj VJ, Chen Y. Nanoparticles - a Review. Trop J Pharm Res 2006; 5(1): 561-573.

3. Allemann E, Gurny R, Doelker E. Drug-loaded nanoparticles - preparation methods and drug targeting issues. Eur J Pharm Biopharm 1993; 39: 173-191.

4. Couvreur $P$, Vauthier $C$. Nanotechnology: intelligent design to treat complex disease. Pharm Res 2006; 23(7): 284-288.

5. Alphandary HP, Andremont $A$, Couvreur $P$. Targeted delivery of antibiotics using liposomes and nanoparticles: research and applications. Int J Antimicrob Agents 2000; 13: 155-168.

6. Schiffelers R, Storm G, Bakker I. Woudenberg. Liposome-encapsulated aminoglycosides in pre-clinical and clinical studies: J Antimicrob Chemother 2001; 48: 333-344.

7. Barratt G. Colloidal drug carriers: achievements and perspectives. Cell Mol Life Sci 2004; 60: 21 37.
8. Bodmeier R, Chen HG, Paeratakul O. A novel approach to the oral delivery of micro- or nanoparticles. Pharm Res 1989; 6: 413-417.

9. Ying-chien $C$, Ya-ping SU, Chiing-chang $C$, Guang JIA, Huey-lan W, Gaston W, Jaung-geng L. Relationship between antibacterial activity of chitosan and surface characteristics of cell wall. Acta Pharmacol Sin 2004 Jul; 25 (7): 932-936

10. Barratt G. Characterization of colloidal drug carrier systems with zeta potential measurements. Pharm Technol Eur 1999; 11: 25-32.

11. Lochmann D, Weyermann J, Georgens $C$, Prassl $R$, Zimmer A. Albumin-protamine-oligonucleotide nanoparticles as a new antisense delivery system. Part 1: Physicochemical characterization. Eur J Pharm Biopharm 2005; 59: 419-429.

12. Zhang X, Xia $Q$, Gu N. Preparation of all- trans retinoic acid nanosuspension using a modified precipitation method. Drug Dev Ind Pharm 2006; 32: 857-863.

13. Moghimi SM, Hunter AC, Murray JC. Longcirculating and target-specific nanoparticles: theory to practice. Pharmacol Rev 2001; 53: 283-318. 